\title{
Theoretical and Experimental Study of Stimulated and Cascaded Raman Scattering in Ultrahigh- $Q$ Optical Microcavities
}

\author{
Tobias J. Kippenberg, Sean M. Spillane, Bumki Min, and Kerry J. Vahala
}

\begin{abstract}
Stimulated Raman scattering (SRS) in ultrahigh- $Q$ (UHQ) surface-tension-induced spherical and chip-based toroid microcavities is considered both theoretically and experimentally. These microcavities are fabricated from silica, exhibit small mode volume (typically $1000 \mu \mathrm{m}^{3}$ ) and possess whispering-gallery type modes with long photon storage times (in the range of $\mathbf{1 0 0}$ ns), significantly reducing the threshold for stimulated nonlinear optical phenomena. Oscillation threshold levels of less than 100 $\mu \mathrm{W}$ of launched fiber pump power, in microcavities with quality factors of $\mathbf{1 0 0}$ million are observed. Using a steady-state analysis of the coupled-mode equations for the pump and Raman whispering-gallery modes, the threshold, efficiencies and cascading properties of SRS in UHQ devices are derived. The results are experimentally confirmed in the telecommunication band (1550 nm) using tapered optical fibers as highly efficient waveguide coupling elements for both pumping and signal extraction. The device performance dependence on coupling, quality factor and modal volume are measured and found to be in good agreement with theory. This includes analysis of the threshold and efficiency for cascaded Raman scattering. The side-by-side study of nonlinear oscillation in both spherical microcavities and toroid microcavities on-a-chip also allows for comparison of their properties. In addition to the benefits of a wafer-scale geometry, including integration with optical, electrical, or mechanical functionality, microtoroids on-a-chip exhibit single mode Raman oscillation over a wide range of pump powers.
\end{abstract}

Index Terms-Cascaded Raman scattering, fiber-coupling, microcavities, stimulated Raman scattering (SRS), tapered optical fiber, ultrahigh- $Q$ (UHQ), whispering gallery modes.

\section{INTRODUCTION}

$\mathbf{U}$ LTRAHIGH- $Q$ (UHQ) surface-tension-induced microcavities (STIMs) combine small modal volume with some of the highest optical quality-factors $(Q)$ to date of nearly 10 billion [1], and are of interest for a variety of studies ranging from fundamental physics such as cavity quantum electrodynamics [2]-[4] to applied areas such as low-threshold and narrow-linewidth lasers [5]-[7], nonlinear optical oscillators [8], [9], as well as high-sensitivity transducers for biochemical sensing [10]. These silica microcavities feature whispering gallery type modes (WGMs) and rely upon exquisite smoothness at the cavity dielectric boundary to attain UHQ performance that is typically in excess of $100 \mathrm{mil}-$ lion. For nonlinear optical studies, strong resonant buildup of energy in microscale volumes significantly reduces the threshold

Manuscript received January 17, 2004; revised August 5, 2004. This work was supported by the National Science Foundation, by Defense Advanced Research Agency, and by the Caltech Lee Center for Advanced Networking.

The authors are with the California Institute of Technology, Pasadena, CA 91125 USA (e-mail: vahala@its.caltech.edu).

Digital Object Identifier 10.1109/JSTQE.2004.837203 for nonlinear optical effects to occur. This was recognized in the pioneering work of Chang [11], [12] and Campillo [13]-[15] who observed and studied a variety of nonlinear optical effects in UHQ liquid microdoplets. Their work used free-space illumination to optically pump the microdroplets and thereby induce Raman oscillation [12], [13], [15], cascaded Raman scattering [12] and Brillouin scattering [16]. Silica UHQ STIMs provide a far more stable and robust microcavity in comparison with liquid microdroplets. However, despite numerous studies on these devices over the past decade [17]-[26] the observation of nonlinear phenomena (beyond thermal effects) in these devices, had been limited to one report on Kerr-induced wavelength shifts at low temperatures [20]. The advent of pumping and signal collection using fiber taper coupling methods [27]-[30] proved an important turning point in access to nonlinear phenomena in this important microcavity system. Fiber tapers provide remarkably efficient coupling to and from the UHQ silica sphere system [28], [30] and enable direct access to the technologically important optical fiber transport medium. The measured fiber-coupled threshold for a variety of nonlinear phenomena in taper-coupled, silica microspheres are lower than for any other nonlinear oscillator reported to date. Silica microsphere Raman lasers with ultralow threshold levels of only $62 \mu \mathrm{W}$ [31] have been demonstrated. Compared to microdroplets these devices allow stable and long term observation of nonlinear optical effects in microcavities. Cascaded Raman lasing in these devices of up to five orders has also been observed [32]. The tapered optical fiber in these experiments functions to both pump WGMs as well as to extract the nonlinear Raman fields. In addition, the tapered-fiber coupling junction is highly ideal [28], making it possible to strongly overcouple UHQ cavities with negligible junction loss. This feature allows for the observation of very high internal differential photon conversion efficiencies approaching unity. Whereas microspheres are both compact and efficient nonlinear oscillators, their fabrication properties lack the control and parallelism typical of microfabrication techniques. Recently-developed UHQ toroid microcavities on-a-chip [33] provide a UHQ silica device with performance equivalent to a microsphere. UHQ toroids have several advantages over spheres including being wafer-scale devices that can be fabricated in parallel as dense arrays or integrated with electronics or other optical functionality. In this paper, we demonstrate and analyze nonlinear Raman oscillation in both microsphere and microtoroid on-a-chip structures. In addition to studying Raman oscillation in microcavities in detail, this work allows us to compare the performance and properties of toroidal and spherical microcavities. In addition to their fabrication and 
integration advantages, it will be seen that microtoroids also have performance advantages in comparison to microspheres. This includes a reduced number of supported azimuthal modes, which allows observation of single-mode Raman oscillation over a large range of pump powers [34] (of critical importance in practical applications), as well as a lower Raman threshold due to a reduced mode volume compared to a spherical cavity.

The paper is organized as follows. The first section will give a brief introduction into the fabrication, coupling, and optical properties of UHQ toroid on-a-chip and spherical microcavities. Section II will present a model for Raman lasing in a waveguide-coupled whispering-gallery-mode resonator. There, we derive the expression for the threshold and the efficiency of the conversion process. In the UHQ regime resonances are easily split into doublets due to intermode coupling of the degenerate clockwise and counterclockwise propagating whispering gallery modes [35]. The effect of this intermode coupling on stimulated Raman scattering is also considered in this section. The case of cascaded Raman oscillation in which Raman signals serve to pump and generate higher-order Raman waves is also treated in the section. In Section IV, the theoretical results are compared with experimental studies of the dependence of Raman threshold on quality-factor, mode volume and waveguide loading. Experimental results concerning power and efficiency during cascaded operation are presented and compared to theory and found to be in good agreement with theoretical predictions. Finally, a comparison of microtoroid oscillation properties with those of microspheres is presented in Section V.

\section{UHQ SURFACE-TENSION-INDUCED MiCROCAVITIES}

Surface-tension-induced microcavities such as microdroplets, exhibit a superb, cavity surface finish (typically nanometer surface roughness [1]) leading to whispering gallery type modes with some of the highest optical quality factors recorded to date. In the work presented here, both spherical and toroid microcavities on-a-chip made from silica are investigated. Both types of structures exhibit $Q$-factors in excess of 100 million. Briefly, the fabrication of both microsphere and microtoroids relies upon surface tension to induce collapse of a given silica preform into the final cavity shape. Since the cavity fabrication involves a temporary liquid state the surface finish of the final "solid" microcavity is excellent. In the case of a microsphere, the preform used here is an optical fiber tip which is heated and melted with a carbon-dioxide laser (10.6 $\mu \mathrm{m}$ wavelength). Surface tension causes the silica fiber tip to contract and form a spherical microcavity, while the remainder of the fiber stem serves as a holder for sphere-positioning. Light within the sphere is confined near an equatorial plane by continuous total internal reflection at the cavity interface. Microtoroids, on the other hand, use wafer microfabrication techniques involving a combination of lithography and etching combined with a final selective reflow process using a $\mathrm{CO}_{2}$ laser. The details of this fabrication process are reported in [33]. In the case of toroid microcavities, the preform consists of a microfabricated silica disk supported by a silicon pillar. Illumination of the disk using a $\mathrm{CO}_{2}$ laser induces selective reflow of the silica. Surface tension causes the disk preform to
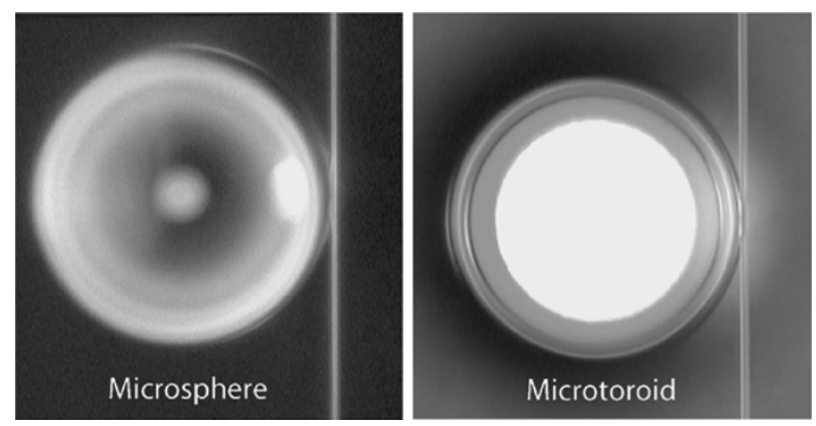

Fig. 1. Optical micrograph of a spherical (left) and toroid microcavity (right) coupled to a tapered optical fiber.

collapse into a toroidal periphery, thereby creating the resonant cavity. Fig. 1 contains an optical micrograph of a microsphere and a microtoroid on-a-chip.

Highly efficient evanescent coupling to these microcavities can be achieved by use of tapered optical fibers [28], [30], which are fabricated by melting and adiabatically tapering a standard, telecommunication fiber until a waist diameter of approximately $1-2 \mu \mathrm{m}$ is reached. When brought into proximity of the WGM resonator and when the taper waist diameter is chosen to phase match to the WGMs, efficient coupling both to and from the microcavity can be achieved. Coupling can be described by the normalized coupling parameter $K=\left(\tau_{0} / \tau_{e x}\right)$ which describes the ratio of intrinsic resonator lifetime $\tau_{0}$ to the external (coupling related) lifetime $\tau_{e x}$. Following the standard conventions [36], undercoupling is denoted by $K<1$, overcoupling by $K>1$, and critical coupling (the point of vanishing waveguide transmission) is denoted by $K=1$. The ability to achieve strong overcoupling is important as it exemplifies the very ideal nature [28] of the taper-microcavity, coupling junction. In addition to efficient excitation and extraction of optical power, the fiber taper provides direct coupling to optical fiber, thereby further facilitating laboratory measurements. In the case of microtoroids on-a-chip, the taper is also crucial as a means of "probing" the whispering gallery devices which are within a few micrometeres of the silicon wafer surface.

Using tapered optical fibers, resonator quality factor can be inferred from either linewidth measurements or cavity ringdown experiments [33]. Fig. 2 shows a cavity ringdown measurement on a 45- $\mu \mathrm{m}$-diameter microtoroid at $1550 \mathrm{~nm}$, exhibiting a critically coupled $Q$ of $10^{8}$. In this measurement, the WGM was excited on resonance, and the fiber-taper adjusted to the critical point. The cavity lifetime can then be inferred by gating-off the excitation laser, and recording the cavity decay signal. The critically coupled $Q$ (including the waveguide coupling contributions) was 100 million, and when correcting the $Q$ for the waveguide loading, as described in Section III-B, an intrinsic quality factor of $3.7 \times 10^{8}$ is inferred.

\section{TheOretical ANALYSis OF RAMAN SCATtERING IN High- $Q$ MicrocaVITES}

\section{A. First-Order Raman Scattering in Microcavities}

Raman scattering in a waveguide coupled microcavity can be described classically by using coupled mode equations for the pump and Raman fields with nonlinear Raman coupling 


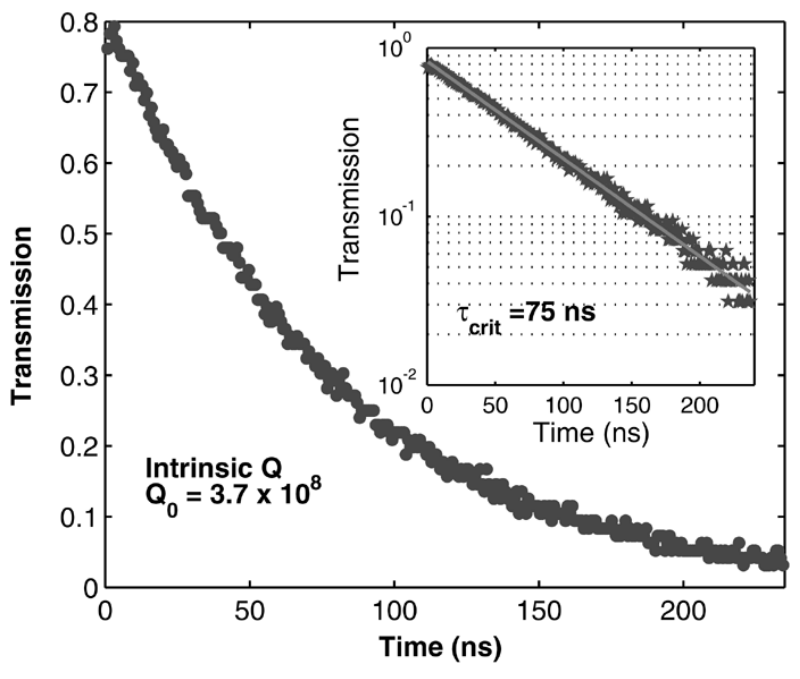

Fig. 2. Cavity rindown measurement of a 50- $\mu$ m-diameter toroid microcavity coupled to a tapered optical fiber. At $t=0$, the laser is gated off, and (after an 8-ns fall-time) the transmission is entirely due to the cavity decay field. Inset: A logarithmic plot to infer the decay time yields $75 \mathrm{~ns}$ at the critical point.

terms. Other nonlinear effects, which can compete with Raman scattering, such as four-wave-mixing or Brillouin scattering are not considered in this analysis as the microcavity poses stringent frequency matching constraints on these processes making their observation difficult. In the case of stimulated Brillouin scattering, the narrow gain bandwidth in the range of $100 \mathrm{MHz}$, makes overlap of cavity modes with the Brillouin gain spectrum unlikely and was not observed in this work. In the case of parametric oscillation mediated by the Kerr-nonlinearity (i.e., four-wave mixing), energy conservation requires a triple resonance condition for signal, idler, and pump mode, which can be satisfied only under certain conditions [37]. These additional constraints for oscillation based on the Kerr nonlinearity or Brillouin gain, cause stimulated Raman scattering-which is intrinsically phase-matched - to be the dominant microcavity nonlinear optical effect. For simplification, we assume that the pump wavelength and the Raman wave are on resonance and use the slowly varying envelope approximation

$$
\begin{aligned}
\frac{d E_{p}}{d t}= & -\left(\frac{1}{2 \tau_{e x}}+\frac{1}{2 \tau_{0}}\right)_{p} E_{p}-\frac{\omega_{p}}{\omega_{R}} g_{R}^{c}\left(\omega_{p}, \omega_{R}\right) \\
& \cdot\left|E_{R}\right|^{2} E_{p}+\kappa s \\
\frac{d E_{R}}{d t}= & -\left(\frac{1}{2 \tau_{e x}}+\frac{1}{2 \tau_{0}}\right)_{R} E_{R}+g_{R}^{c}\left(\omega_{p}, \omega_{R}\right) \\
& \cdot\left|E_{P}\right|^{2} E_{R} .
\end{aligned}
$$

Here, $E$ signifies the slowly-varying amplitude of the pump and Raman WGM modes of the cavity and $s$ denotes the input wave. The excitation frequency of the pump mode and resonant Raman mode is given by $\omega_{R}$ and $\omega_{p}$ and $\tau$ is the total lifetime of photons in the resonator, which is related to the quality factor by $Q=\omega \cdot \tau$. The coupling coefficient $\kappa$ denotes the coupling of the input pump wave $s$ to the cavity whispering-gallery-mode $E_{p}$. The relation $\kappa=\sqrt{1 / \tau_{e x}}$ associates the coupling coefficient with a corresponding lifetime, such that $1 / \tau=\left(1 / \tau_{e x}\right)+\left(1 / \tau_{0}\right)$ [36]. Since the Raman effect will excite both eigenmodes of the cavity (clockwise and counterclockwise propagating modes), equal amplitude emission occurs along both waveguide directions given by $\left|s_{r}\right|^{2}=\left(\left|E_{R}\right|^{2} / 2 \tau_{e x}\right)$. The Raman intracavity gain coefficient is denoted as $g_{R}^{c}$, which can be related to the more commonly used gain coefficient $g_{R}$ (measured in units of meters per Watt) by

and

$$
g_{R}^{c} \equiv \frac{c^{2}}{2 n^{2}} \frac{1}{V_{\mathrm{eff}}} g_{R}
$$

$$
V_{\text {eff }}=\frac{\int\left|\vec{E}_{P}\right|^{2} d V \int\left|\vec{E}_{R}\right|^{2} d V}{\int\left|\vec{E}_{P}\right|^{2}\left|\vec{E}_{R}\right|^{2} d V}
$$

where $V_{\text {eff }}$ is the effective modal volume [38], and $\vec{E}$ is the electric field vector [2]. The effective mode volume accounts for the intensity dependent gain, and for silica microspheres and microtoroids has approximately twice the value than the energy related definition of mode volume. Steady-state analysis of the coupled mode equations, results in a clamped cavity pump field above threshold. This clamping alters the coupling of pump power to the resonator, and, in turn, the pump power dependence of Raman laser power such that the following square root dependence results

$$
P_{R}=\frac{\omega_{r}}{\omega_{p}}\left(\frac{1}{\tau_{e x}}\right)^{2}\left(\frac{1}{2 \tau_{0}}+\frac{1}{2 \tau_{e x}}\right)^{-2} \cdot P_{t}\left(\sqrt{\frac{P}{P_{t}}}-1\right) .
$$

The physical origin of this square root dependence of the pump-to-Raman conversion can be viewed as a "pumping inefficiency," i.e., the coupled pump power does not increase linearly with launched fiber power. The nonlinear dependence of coupled pump power can be illustrated by considering a pump wave that is initially critically coupled to the resonator. As noted earlier, critical coupling features complete transfer and dissipation of power from the resonator (i.e., zero transmission). In terms of the fields involved in coupling both to and from the resonator, critical coupling results from the destructive interference of the cavity leakage field with the transmitted, pump field (i.e., the portion that does not couple to the resonator from the waveguide). Once the onset of Raman lasing is reached, the cavity pump field is clamped at the threshold value resulting in a fixed cavity pump leakage field. Subsequent increase in launched pump power will imbalance the leakage and the transmitted pump fields, giving rise to finite transmission and a shift away from the critical point. The pump coupling to the resonator is thereby less and less efficient as the pump field is increased. The expression for the Raman threshold pump power can be factorized into terms involving modal volume, waveguide-cavity coupling strength and cavity lifetime (or quality factor). To facilitate separation of the coupling and intrinsic lifetime dependence, we use the dimensionless normalized coupling parameter $K_{p}=\left(\tau_{0} / \tau_{e x}\right)_{p}$. In the ideal case of a single-mode waveguide coupled to a whispering-gallery-mode the waveguide transmission as a function of coupling is given by $T=(1-K / 1+K)^{2}$ and $K$ typically varies exponentially with the waveguide-microcavity "coupling gap" distance [36]. Using these definitions and under the assumption of equal coupling properties and photon lifetimes for both the pump and 
Raman mode, i.e., $K_{p}=K_{R} \equiv K$ and $\tau_{R}=\tau_{p}$, the threshold expression is given by

$$
P_{t}=C(\Gamma) \frac{\pi^{2} n^{2}}{g_{R} \lambda_{p} \lambda_{R}} V_{\text {eff }} \cdot\left(\frac{1}{Q_{0}}\right)^{2} \cdot \frac{(1+K)^{3}}{K} .
$$

Here, we have also introduced $C(\Gamma)$ which is a possible correction factor to account for intermode coupling of the degenerate clockwise and counterclockwise propagating whisperinggallery modes. This factor will be explained in Section III-B. The threshold expression follows an inverse square dependence on the quality factor. This reflects the fact that an increase in $Q$ will cause a twofold benefit in terms of both reducing cavity round trip losses that must be overcome for threshold as well as increasing the Raman gain, due to the intensity dependence of the Raman gain coefficient on the pump field. In addition, the equation shows that the threshold scales linearly with the modal volume. Both the coupling and mode volume dependence of the Raman threshold are examined experimentally in Section III-B. When analyzing the coupling dependence under the assumption of equal Raman and pump quality factors and coupling factors, the minimum threshold occurs when $Q_{e x}^{\min }=2 \cdot Q_{0}$ or $K^{\mathrm{min}}=(1 / 2)$, i.e., in the undercoupled regime with finite waveguide transmission of $T^{\min }=(1 / 9)(\sim 11 \%)$. This minimum pump threshold is given by

$$
P_{t}^{\min }=C(\Gamma) \frac{\pi^{2} n^{2}}{g_{R} \lambda_{p} \lambda_{R}} V_{\text {eff }} \cdot\left(\frac{1}{Q_{0}}\right)^{2} \cdot \frac{27}{4} .
$$

It is worth noting that at this coupling condition, the circulating pump-power in the resonator is not maximum. This can be understood since minimum threshold represents an optimal balance of both pump coupling and Raman mode coupling loss. The conversion of pump power to Raman power above threshold can be characterized by the differential slope efficiency. The bidirectional external differential slope efficiency $\eta_{e x}$ is derived by linearizing the expression for $P_{R}$ near the threshold condition and is given by

$$
\eta_{\text {ex }} \equiv \frac{d P_{\text {Raman }}}{d P_{\text {launched }}}=2 \cdot \frac{\omega_{R}}{\omega_{p}}\left(1+\frac{1}{K}\right)^{-2} .
$$

Fig. 3 shows the differential slope efficiency as a function of coupling strength. It is noteworthy that it approaches the value of $2\left(\omega_{R} / \omega_{p}\right)$ in the limit of strong overcoupling $\left(\left(\tau_{0} / \tau_{e x}\right)=\right.$ $\infty)$. Surprisingly, this value exceeds unity, indicating that every waveguide pump photon added above threshold, is converted to more than one Raman photon. This result can be understood by again considering the nonlinear dependence of coupled pump power, except this time in the over-coupled regime. In particular, the differential increase in coupled pump power grows more quickly in the overcoupled (more slowly in the undercoupled regime) than the differential increase in launched pump power. This leads to the interesting effect that the differential photon conversion efficiency can exceed unity. Taking into account the nonlinear dependence of coupled pump power by defining the internal differential efficiency $\eta_{\text {int }}$ as the coupled (as distinct from the launched) pump-to-Raman power, the efficiency ap-

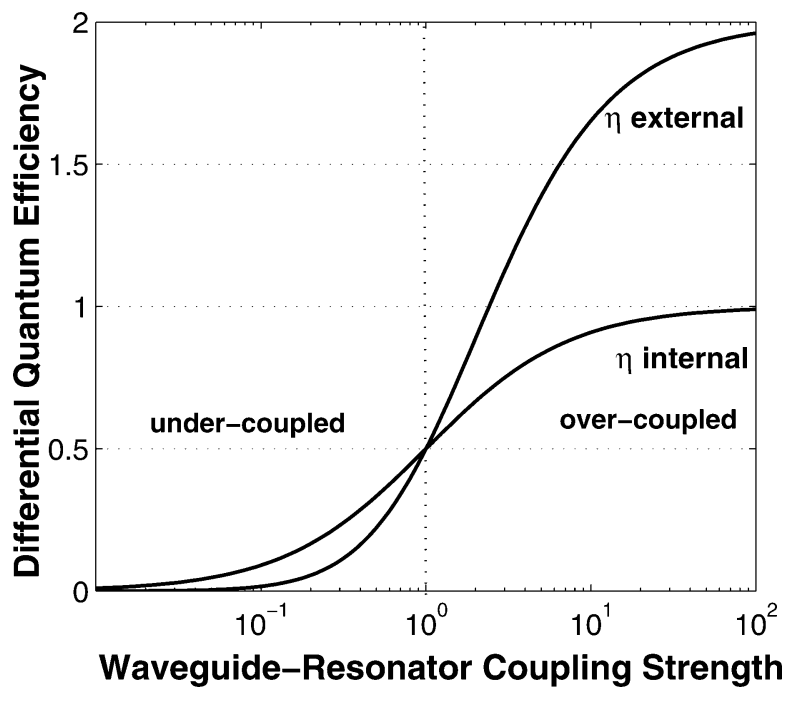

Fig. 3. External and internal pump to Raman differential conversion efficiency. The external efficiency refers to conversion of launched pump power to Raman output power, while the internal efficiency is the coupled pump power to raman output power. As evident, the external efficiency can exceed unity in the overcoupled regime.

proaches, as expected, the value $\omega_{R} / \omega_{p}$ in the limit of strong overcoupling

$$
\eta_{\text {int }} \equiv \frac{d P_{\text {Raman }}}{d P_{\text {coupled }}}=\frac{\omega_{R}}{\omega_{p}}\left(1+\frac{1}{K}\right)^{-1} .
$$

Fig. 3 shows both the internal and external differential Raman conversion efficiencies as a function of coupling strength.

\section{B. Effect of Intermode Coupling on Stimulated Raman Scattering}

In the UHQ regime, resonances of a whispering-gallery-type microcavity are often split into doublets [17], [35]. This splitting is due to coupling of the degenerate clockwise and counterclockwise propagating modes by either intrinsic or surface scattering centers. The modified coupling properties have been extensively studied [39]. Here, we briefly summarize the results of this study and analyze the effect of intermode coupling on Raman scattering. The extent to which intermode coupling modifies the waveguide coupling properties can be described by the dimensionless intermode coupling parameter $\Gamma=$ $\left(\tau_{0} / \gamma\right)$, where $1 / \gamma$ is the rate of coupling of the degenerate clockwise and counterclockwise modes. It is easily measured as the linewidth normalized splitting in the undercoupled regime and essentially gives a measure for the visability of the doublet structure (see Fig. 4, inset). The presence of intermode coupling has several consequences. First, the critical point (as defined by vanishing waveguide transmission) is shifted toward a coupling point, which, using the conventional coupling terminology, is considered overcoupled as $\tau_{e x}<\tau_{0}$. The point of vanishing transmission occurs at

$$
K_{\mathrm{crit}}=\sqrt{\Gamma^{2}+1} .
$$

Second, the shifted critical point is accompanied by a maximum reflection into the backward direction of the waveguide (i.e., 


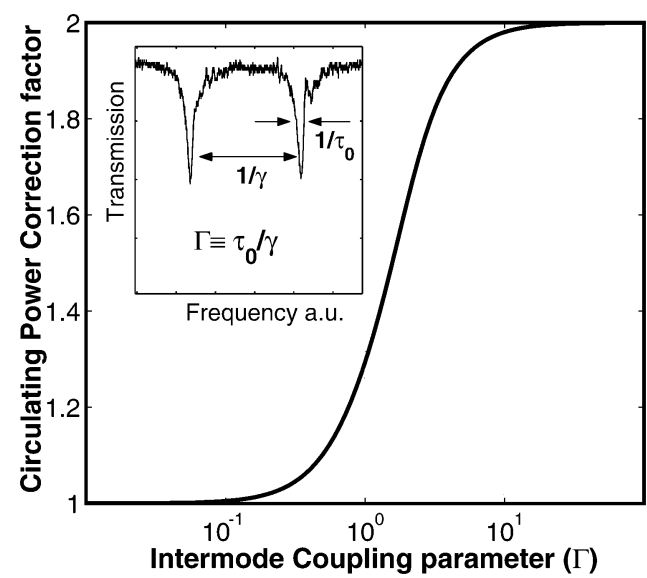

Fig. 4. Power correction factor (which is the ratio defined by maximum circulating power in the absence and presence of modal coupling) as a function of $\Gamma$-parameter. The inset shows a frequency scan of a doublet of an UHQ mode.

the contra-directional waveguide mode). The magnitude of the reflection at the previously modified critical point is given by

$$
R^{\max }=\left(\frac{\Gamma}{\sqrt{\Gamma^{2}+1}+1}\right)
$$

Third, the leakage of the cavity field into the backward waveguide direction causes a reduction of the cavity buildup factor with respect to the ideal case in the absence of intermode coupling. In the limit of strong modal coupling, the cavity buildup factor is reduced by a factor of 2 , which subsequently causes a twofold increase in the threshold necessary to achieve Raman lasing. Fig. 4 shows the circulating power correction factor $C(\Gamma)$ as a function of the dimensionless intermode coupling parameter $\Gamma$. In the presence of modal coupling, the waveguide coupling condition for minimum threshold experiences a slight shift toward overcoupling with the maximum shift occurring at $\Gamma \approx 1.52$. In the regime of very strong modal coupling, the condition of minimum Raman threshold approaches again the original condition $K=1 / 2$.

\section{Analysis of Cascaded Raman Scattering in High-Q Microcavities}

The first Raman field can itself act as a secondary pump field and generate further Raman modes. This process of cascaded Raman scattering can be described by including higher order coupling terms into the coupled mode equations of pump and Raman fields as shown in the following [32]:

$$
\begin{aligned}
\frac{d E_{p}}{d t}= & {\left[-\left(\frac{1}{2 \tau_{t}}\right)_{P}-g_{R 1}^{c}\left(\frac{\omega_{P}}{\omega_{R}}\right)\left|E_{R 1}\right|^{2}\right] E_{p}+\sqrt{\frac{1}{\tau_{e x}}} s } \\
\frac{d E_{R 1}}{d t}= & {\left[-\left(\frac{1}{2 \tau_{t}}\right)_{R 1}+g_{R 1}^{c}\left|E_{p}\right|^{2}-g_{R 2}^{c}\left(\frac{\omega_{R 1}}{\omega_{R 2}}\right)\left|E_{R 1}\right|^{2}\right] } \\
& \times E_{R 1} \\
\frac{d E_{R 2}}{d t}= & {\left[-\left(\frac{1}{2 \tau_{t}}\right)_{R 2}+g_{R 2}^{c}\left|E_{R 1}\right|^{2}-g_{R 3}^{c}\left(\frac{\omega_{R 2}}{\omega_{R 3}}\right)\left|E_{R 3}\right|^{2}\right] } \\
& \times E_{R 2} \cdots \\
\frac{d E_{R N}}{d t}= & {\left[-\left(\frac{1}{2 \tau_{t}}\right)_{R N}+g_{R N}^{c}\left|E_{R(N-1)}\right|^{2}\right] E_{R N} }
\end{aligned}
$$

where $N$ is the Raman order. To find the corresponding thresholds and output powers for these higher order processes, the set of equations can be solved iteratively in steady state. Here, we have introduced the dimensionless coefficients $c_{i}$

$$
c_{i} \equiv \frac{\omega_{i}}{\omega_{i+1}} \cdot \frac{g_{i+1}^{c}}{g_{i+2}^{c}}=\frac{\omega_{i}}{\omega_{i+1}} \cdot \frac{V_{\text {eff }}\left(\lambda_{i+2}\right)}{V_{\mathrm{eff}}\left(\lambda_{i}\right)} .
$$

The general solutions for the threshold of the even and odd order $N$ th Raman modes are given by the following expressions. As in the previous section, we have assumed equal coupling strengths and intrinsic $Q$ factors for the pump and Raman modes

$$
\begin{aligned}
P_{t}^{N=2 m} & =\frac{1}{g_{R}^{c}} \frac{\tau_{e x}}{\left(\tau_{t}\right)^{3}}\left(\sum_{i=0}^{m}\left(c_{i}\right)^{i}\right)^{2}\left(\sum_{i=0}^{m-1}\left(c_{i}\right)^{i}\right) \\
P_{t}^{N=2 m+1} & =\frac{1}{g_{R}^{c}} \frac{\tau_{e x}}{\left(\tau_{t}\right)^{3}}\left(\sum_{i=0}^{m}\left(c_{i}\right)^{i}\right)^{3} .
\end{aligned}
$$

As evident from these expressions, even- and odd-order stokes fields exhibit different threshold powers as a function of stokes order $(N)$. When considering Raman scattering in silica at optical frequencies, one can approximate the earlier expressions by taking $c_{i} \approx 1$ since the Raman shift is small compared to the optical frequency. In addition, it is assumed that the mode volume is wavelength independent. Under this assumption, the threshold expressions reduce to

$$
\begin{aligned}
P_{t}^{N=2 m+1} & =C(\Gamma) \frac{\pi^{2} n^{2}}{g_{R} \lambda_{p} \lambda_{R}} V_{\text {eff }} \frac{1}{Q_{0}^{2}} \frac{(1+K)^{3}}{K} \cdot \frac{(N+1)^{3}}{8} \\
P_{t}^{N=2 m} & =C(\Gamma) \frac{\pi^{2} n^{2}}{g_{R} \lambda_{p} \lambda_{R}} V_{\text {eff }} \frac{1}{Q_{0}^{2}} \frac{(1+K)^{3}}{K} \cdot \frac{N(N+2)^{2}}{8} .
\end{aligned}
$$

It follows that the threshold for cascaded Raman oscillation exhibits a cubic dependence on Raman order $N$. The emission power dependences vary depending upon whether the highest order wave is even or odd. For the odd-order case, all odd orders increase as the square root of the pump power and even orders are clamped. For the even-order case, all even-order lines increase linearly with pump power while odd orders are clamped. Fig. 5 illustrates this behavior showing the Raman output for several stokes orders as a function of input pump power. The analytic expressions for the Raman output power in these cases are given by

$$
\begin{aligned}
P^{N=2 m+1} & =\eta_{e x}^{N} \cdot 2\left(\sqrt{P_{t}^{N} P}-P_{t}^{N}\right) \\
P^{N=2 m} & =\eta_{e x}^{N} \cdot\left(P-P_{t}^{N}\right) .
\end{aligned}
$$

The differential power conversion efficiencies can be obtained by linearizing the previous expressions near the threshold condition. The external and internal differential efficiencies decrease steadily as a function of stokes order $(N)$. For optical frequencies that are much larger than the Raman shift, the external differential conversion efficiency reduces to

$$
\begin{aligned}
\eta_{e x}^{N=2 m} & =\frac{\lambda_{p}}{\lambda_{R N}}\left(1+\frac{1}{K}\right)^{-2} \cdot \frac{16}{(N+2)^{2}} \\
\eta_{e x}^{N=2 m+1} & =\frac{\lambda_{p}}{\lambda_{R N}}\left(1+\frac{1}{K}\right)^{-2} \cdot \frac{8}{(N+1)^{2}} .
\end{aligned}
$$




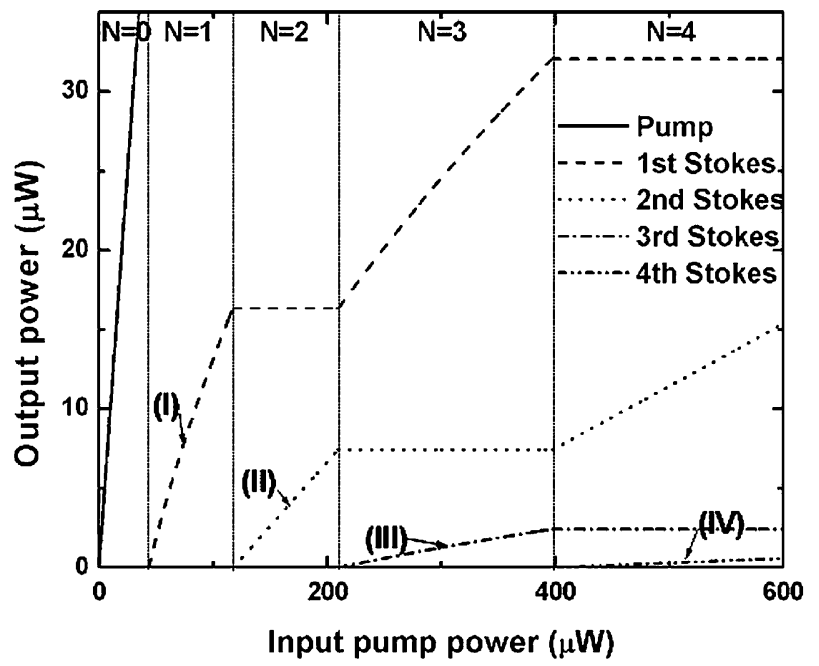

Fig. 5. Theoretical plot of cascaded Raman output power versus pump power for up to fourth order stokes power. Even- and odd-ordered Raman modes exhibit alternating clamping behavior.

For high-order $(N)$ Raman fields, the external differential conversion efficiency thus follows a $1 / N^{2}$-dependence.

\section{EXPERIMENTAL STUDY OF STIMULATED RAMAN SCATTERING IN UHQ MICROSPHERES}

We have observed stimulated Raman scattering using fiber-taper-coupled, UHQ, surface-tension-induced silica microspheres. Tapered optical fibers provide both a very efficient and practical means for pumping and laser signal extraction through the same fiber. The experimental setup in this study is identical to the one reported earlier [31] and the tapered fiber was attached to a piezoelectric stage which allowed precise control of the taper-microcavity coupling gap (20-nm resolution) and variation of the waveguide-resonator coupling strength. The $Q$-factors obtained in the microspheres were typically in the range of $1-2 \times 10^{8}$ and taper insertion loss (fiber to fiber) was usually less than $5 \%$.

To excite the UHQ modes, we used a narrow-linewidth, external-cavity laser emitting in the $1550-\mathrm{nm}$ band. In the case of microspheres, the WGM field spatial forms are known analytically and characterized by the radial, angular, azimuthal, and polarization mode number $(n, \ell, m, p)$. Due to fabrication-induced eccentricity, the $(2 \ell+1)$-fold-degeneracy of the azimuthal modes is lifted yielding a complex mode spectrum. For the spheres considered in this work the eccentricity-induced splitting was in the range of several gigahertz. Microtoroids on-a-chip, on the other hand, possess a significantly reduced mode spectrum due to the strong azimuthal modal confinement provided by the toroid geometry. This both simplifies their spectra and enables operation of microtoroid-Raman lasers in the desirable single mode regime, which is of significant practical importance.

Stimulated Raman oscillation was observed by pumping a single WGM and monitoring the transmission using an optical spectrum analyzer. Once the threshold for SRS was exceeded, lasing modes in the 1650 -nm band could be observed, in correspondence with the peak Raman gain which occurs downshifted in frequency by approximately $14 \mathrm{THz}$ relative to the

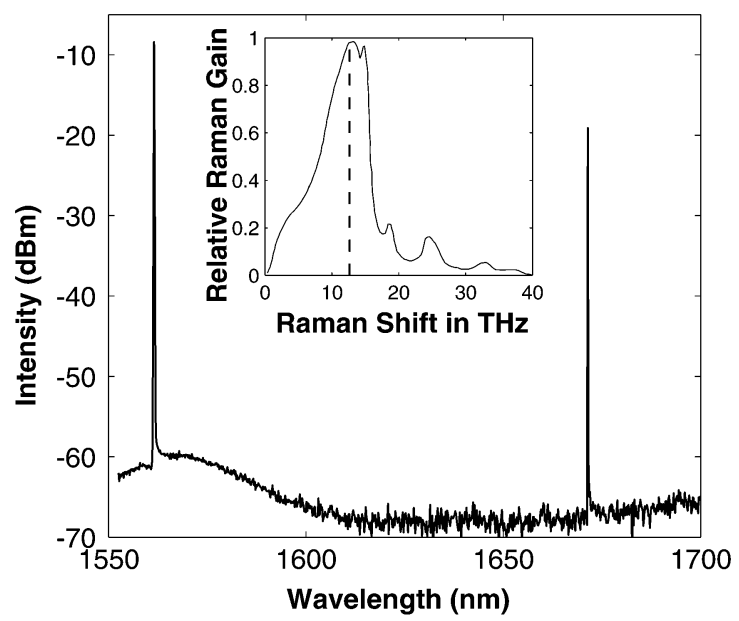

Fig. 6. Single mode Raman lasing in a UHQ spherical microcavity. The pump wavelength is located at $1550 \mathrm{~nm}$ and Raman lasing appears at $1660 \mathrm{~nm}$. The inset shows the Raman frequency shift (designated by the dotted line) overlaid with the silica Raman gain spectrum.

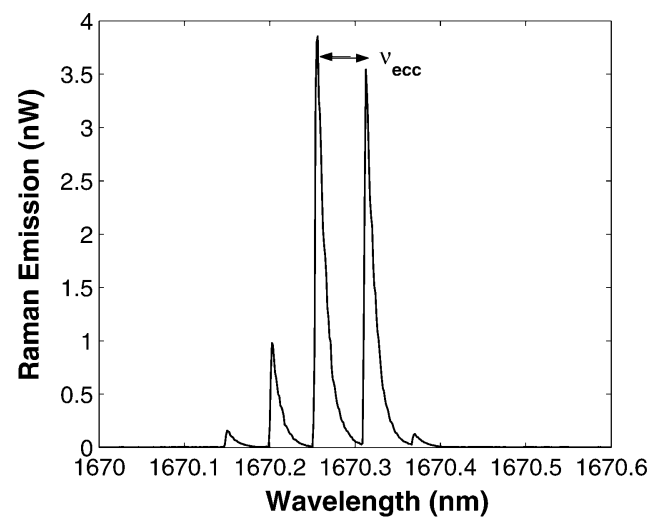

Fig. 7. Spectrally resolved Raman emission of the UHQ microsphere shown in the previous figure, revealing that five eccentricity split azimuthal modes were oscillating simultaneously. The pump power in this measurement was adjusted to slightly above Raman oscillation threshold. The asymmetric line-shape of the Raman modes is caused by repeatedly scanning the pump laser through the pump resonance, which causes a redshift of the cavity resonances, due to heating and subsequent expansion of the cavity (thermal bistability as noted in [17]).

pump frequency (wavelength shift of approximately $110 \mathrm{~nm}$ ). Fig. 6 shows Raman emission for an UHQ microsphere. The Raman emission with respect to the gain peak is provided in the inset of Fig. 6. Since the fundamental whispering gallery modes $(n=1, \ell=m, p=T M)$ are most tightly confined (i.e., smallest mode volume), Raman lasing is expected to occur first for these modes. The presence of nearly degenerate azimuthal modes in a spherical microcavity (i.e., weak eccentricity splitting), causes simultaneous oscillation on several azimuthal modes. Fig. 7 shows a higher resolution spectral scan of the spectrum in Fig. 6. Several azimuthal modes can be observed to be oscillating simultaneously.

The threshold formula predicts a strong dependence of the Raman threshold on waveguide coupling. Fig. 8 shows the measured Raman threshold as a function of taper-microcavity gap distance for a fundamental WGM. The solid line is a fit using the theoretical results from the first section (3) and assuming equal pump and Raman $Q$ factors and coupling conditions. The minimum threshold does indeed occur undercoupled with finite 


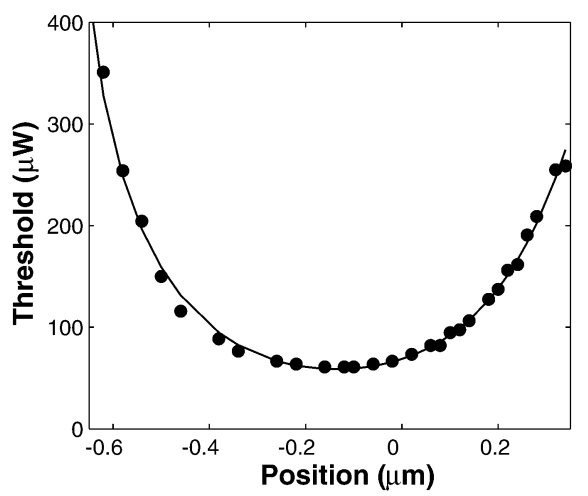

Fig. 8. Dependence of Raman threshold on loading as evidenced by the taper-micro-cavity gap distance. The taper-microcavity gap is measured in microns with respect to the critical point (defined as vanishing taper transmission), and positive separation corresponds to overcoupling. The minimum threshold is $62 \mu \mathrm{W}$ of launched pump power (measured before the taper which includes the insertion loss of the optical fiber taper). Minimum threshold occurs ca. $120 \mathrm{~nm}$ undercoupled (corresponding to a transmission of $13 \%)$.

taper transmission at $T=13 \%$ in good agreement with the theoretically predicted value of $T=1 / 9$.

As a further verification of the threshold formula, we compared the theoretical minimum threshold value with the observed value. The quality factor and the mode splitting of the whispering-gallery mode were measured by performing a linewidth sweep in the undercoupled regime, where the backscattering-induced doublet structure is most pronounced. These measurements yielded $Q_{0}=1 \times 10^{8}$ and $\Gamma=2$. The size of the microsphere was inferred from the free spectral range, $\Delta \lambda=10.5 \mathrm{~nm}$ i.e., $50-\mu \mathrm{m}$ diameter, where the free-spectral range denotes here modes with successive angular mode number $\ell$. The mode volume was calculated using analytic expressions based on estimated mode numbers for the fundamental WGM $(n=1, p=T M, m=\ell, \ell \approx 139)$. Calculations [40], [41] yielded a modal volume of ca. $1300 \mu \mathrm{m}^{3}$. The overlap factor for pump and Raman mode is assumed to be unity. Using these values, the theoretically expected minimum threshold is given by $50 \mu \mathrm{W}$ which is in good agreement with the experimentally measured value of $62 \mu \mathrm{W}$.

The dependence of the Raman threshold on the modal volume was also investigated. For this measurement the Raman threshold was measured for microsphere resonators having varying diameters in the range of ca. $25-120 \mu \mathrm{m}$. (For diameters smaller than $25-\mu \mathrm{m}$ thermally induced wavelength shifts lead to pumping instabilities.) In this diameter range, the mode volume follows an approximately quadratic dependence (actual inferred exponent is $V \propto R^{1.83}$ [40], [41]) on the sphere radius. As an aside, for smaller spheres the mode volume deviates from this behavior and ultimately, for very small diameters, the mode volume increases due to weakening of the whispering gallery confinement [40]. The minimum mode volume occurs for a radius of $6.9 \mu \mathrm{m}$ (for $\ell=m=34$ ) [40] for $1550 \mathrm{~nm}$ wavelength and the mode volume is $V_{\min }=173.1 \mu \mathrm{m}^{3}$. However, this size is not optimum for stimulated Raman scattering as the additional benefit of reduced mode volume is more than offset by the significant decrease in $Q$ factor to $10^{5}$ [41] (threshold power $\propto V / Q^{2}$ ). For small mode volumes, it has been predicted that the gain coefficient can exhibit a dependence on mode volume

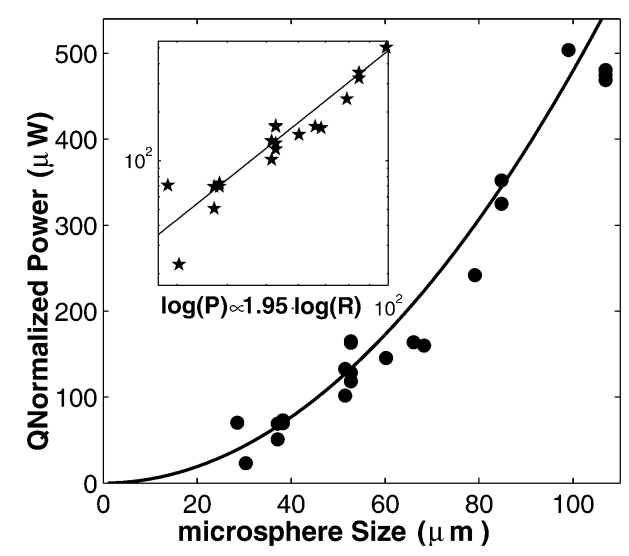

Fig. 9. Raman oscillation pump threshold of a spherical UHQ microcavity as a function of microsphere diameter. To compare microspheres with different resonance characteristics, the Raman threshold was $Q$-normalized to $Q=1.0 \times 10^{8}$ and modal coupling normalized to $\Gamma=0$. The inset shows a double logarithmic plot of the data. A linear fit obtains a dependence of $R^{1.95}$ which is in close agreement with the theoretically expected dependence of $R^{1.83}$.

due to cavity quantum electrodynamics (cQED) effects [14]. However, in the case of stimulated Raman scattering in silica microspheres these effects are not expected to be observable [42] and an approximately quadratic relationship due to the mode volume is predicted.

Fig. 9 shows the experimental results for threshold versus microsphere diameter. In this experiment, the minimum Raman threshold $P_{t}^{\min }$, the microsphere size (as inferred from the free-spectral-range), the intrinsic $Q$ in the pump band $\left(Q_{0}\right)$ and the intermode coupling parameter $(\Gamma)$ were measured. To extract the volumetric dependence of the Raman threshold using data from cavities having different resonant characteristics, the threshold data were normalized to the set of parameters $\left(Q_{0}=10^{8}, \Gamma=0, g_{R}=g_{R}^{\max }\right)$. The result of this procedure is shown in Fig. 9. The data indeed show a quadratic dependence on $R$ (the actual fitted exponential from a double logarithmic plot is 1.95 and is in good agreement with the expected value of 1.83) and confirm the linear relationship of the Raman threshold on the mode volume as predicted by (4).

\section{A. Cascaded Raman Scattering in UHQ Microspheres}

In addition to first-order Raman scattering, we have also observed cascaded Raman scattering. Fig. 10 shows a typical cascaded Raman spectrum, for a UHQ microcavity pumped at 1450 $\mathrm{nm}$. The pump-to-Raman conversion characteristics for firstorder Raman scattering and the second-order Raman mode are shown in the inset. It can be seen that the first-order mode does indeed exhibit a square-root dependence on the launched pump power. The solid line is a fit using (12). The higher order Raman mode exhibits the expected linear increase with pump power.

To study cascaded Raman scattering beyond second-order, experiments using a 980-nm wavelength pump [32] were employed. The shorter wavelength pump allowed the observation of up-to fifth-order cascades (from 980-1300 nm) owing to the reduced mode volume at shorter wavelengths, and the higher Raman gain coefficient $\left(g_{R} \propto 1 / \lambda\right)$. With less than $900 \mu \mathrm{W}$ of launched fiber power up to fifth-order Stimulated Raman Scattering was observed [32], and the threshold and efficiency 


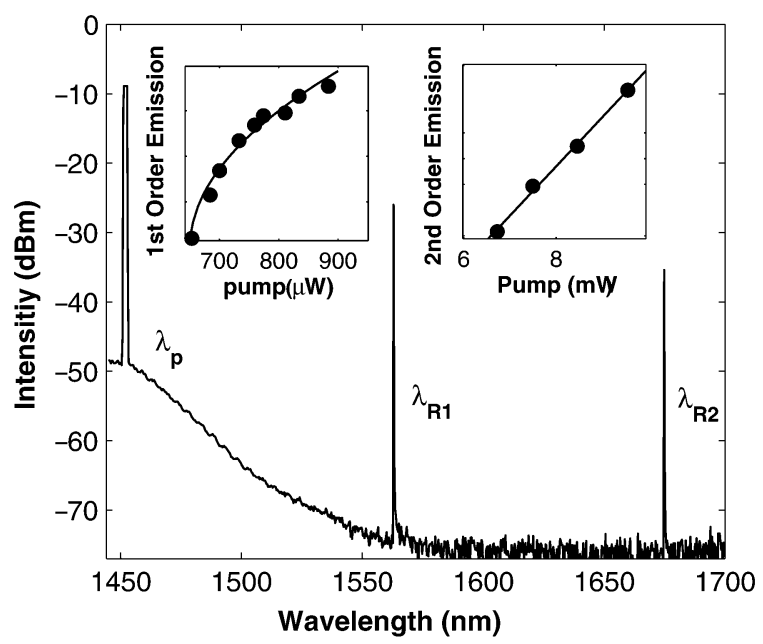

Fig. 10. Cascaded Raman scattering in a 58- $\mu$ m-diameter microcavity. The pump WGM is located at around $1450 \mathrm{~nm}$. The insets show the pump-to-Raman conversion for first- (left inset) and second-order (right inset) Raman modes (measured on different microcavities). The first-order Raman mode exhibits a square-root, and the second-order Raman mode a linear pump-to-Raman conversion characteristic, in agreement with the theoretical prediction. Solid lines: a theoretical fit using (12).

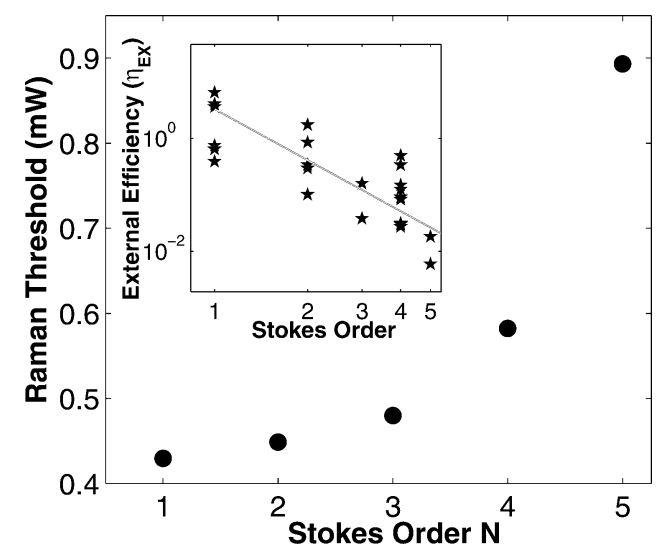

Fig. 11. Fifth-order cascaded Raman scattering. The threshold of the cascades are plotted as a function of stokes order $(N)$. The data was acquired on a single microsphere of 50- $\mu$ m-diameter excited using a WGM at $980-\mathrm{nm}$ wavelength. The inset shows the efficiency of the cascades as a function of cascading order, measured on several devices.

of the cascades measured. Fig. 11 shows the Cascaded Raman threshold for a microsphere as a function of the order of the cascade, for fixed coupling condition. The solid line is a fit [as predicted by (15)], which yields good qualitative agreement with the experimentally measured efficiencies. The inset of Fig. 11 shows a double logarithmic plot of the measured differential conversion efficiency of the cascaded Raman scattering process. The efficiency of the cascades decreases as a function of Stokes order, as is theoretically predicted by (13).

\section{RAMAN SCATTERING IN UHQ TOROID MICROCAVITIES ON-A-CHIP}

Toroid microcavities allow on-chip integration of UHQ performance with other optical, mechanical and electrical functionality. In addition, the wafer-scale fabrication process allows precise dimensional control and parallelism. Fig. 12 shows a scanning electron microscope of a toroid microcavity. In addition to the design freedom and control brought about

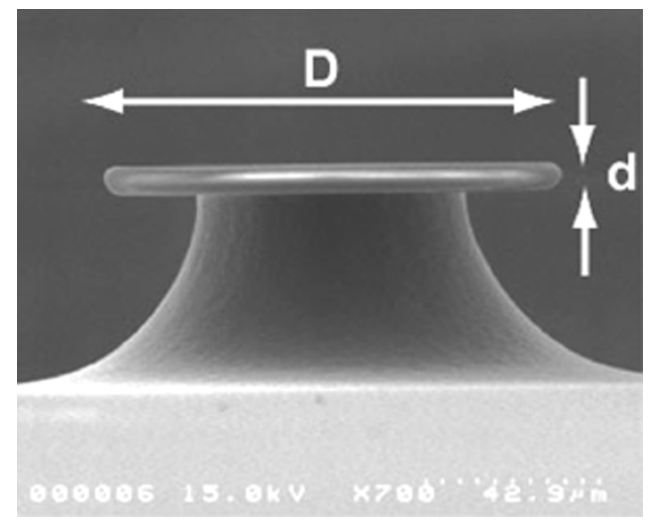

Fig. 12. Scanning-electron-micrograph of a toroid microcavity. The principal diameter $(D)$ and minor toroid diameter $(d)$ are shown.

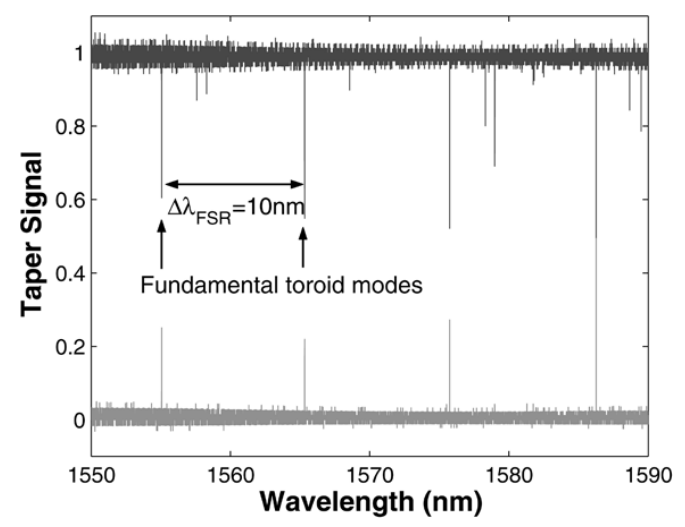

Fig. 13. Recorded mode spectrum (both transmission and reflection) of a 50- $\mu \mathrm{m}$-diameter toroid microcavity, exhibiting a strongly reduced mode spectrum. The reduced density of modes is due to the increased azimuthal confinement, and is similar to spectra obtained with spheroids [26].

by micro-fabrication and integration possibilities, toroid microcavities also exhibit significant advantages in terms of their Raman-emission properties. Whereas microsphere cavities show low threshold operation, their emission is inherently multimode due to the presence of azimuthal modes, as shown in Fig. 7 of the previous section. In contrast, toroid microcavities on-a-chip have a significantly reduced mode spectrum, such that single mode Raman lasing can be observed. The reduced number of azimuthal modes is due to the cavity geometry, which supports only a few modes in the azimuthal direction due to the toroidal confinement [26]. Fig. 13 shows the mode spectrum of a toroid microcavity. Only two higher order modes are present in the spectrum, and successive modes are separated by the free spectral range of the cavity, which in the figure is 10 $\mathrm{nm}$. This is in contrast to a microsphere of identical principal diameter which would support $2 \ell+1$ azimuthal modes weakly split by eccentricity.

The suppression of azimuthal modes, has important consequences on the spectral emission properties of single and cascaded Raman scattering. Most notably, single transverse mode Raman oscillation can be observed over a large range of pump powers. Fig. 14 shows a toroid microcavity Raman laser, which exhibits single mode oscillation at emission power levels up to $160 \mu \mathrm{W}$ with high efficiency (45\% at the critical point). The single-mode emission property is a significant advantage in practical applications of nonlinear optical oscillators. Toroidal 


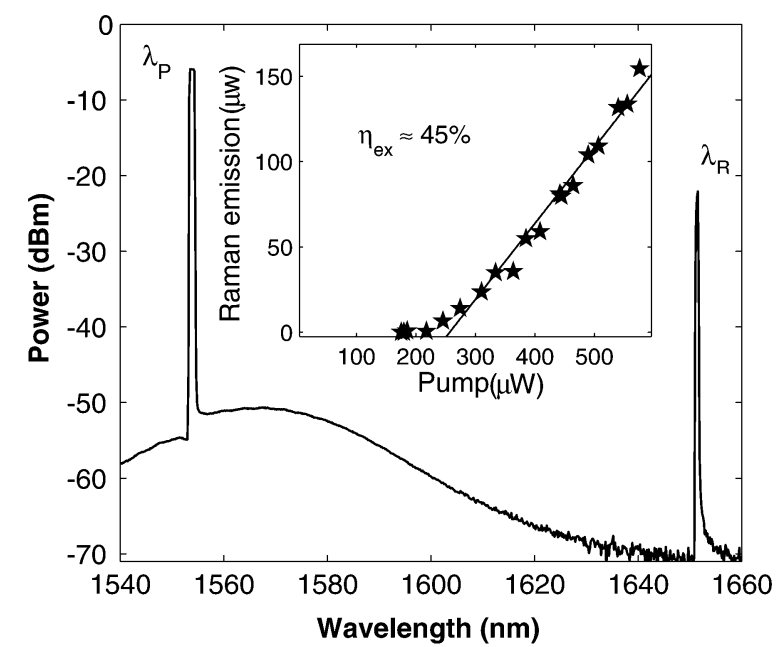

Fig. 14. Single mode Raman emission of a ca. $60-\mu$ m-diameter toroid microcavity. The pump is located at $1550 \mathrm{~nm}$.

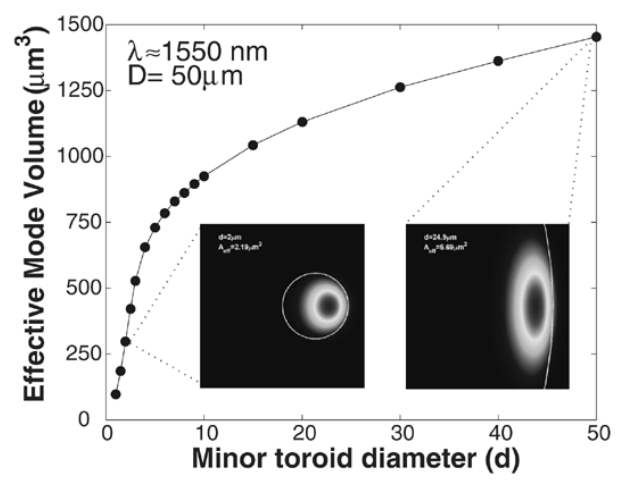

Fig. 15. Numerically calculated effective mode volume of a toroid microcavity as a function of the cross-sectional diameter of the toroid (and fixed principal toroid diameter $D=50 \mu \mathrm{m}$ ). The circles denote the numerically calculated effective mode volume for a fundamental toroid WGM in the 1550-nm band for (TE) polarization. The inset shows the modeled TE-mode intensity distribution for a toroid with $2 \mu \mathrm{m}$ and $50 \mu \mathrm{m}$ toroid-cross sectional diameter $(d)$ using the finite-element method.

and spherical microcavities having similar $Q$ and outer diameter will differ in modal volume. Toroids, owing to the added transverse confinement will have a reduced mode volume and, hence, a lower Raman threshold. The degree to which the threshold is reduced will depend on the "aspect ratio" of the toroid or $D / d$ where $D$ is the outer or principal diameter and $d$ is the minor diameter (see Fig. 12). The modal volume of the fundamental toroid mode in a $D=50 \mu \mathrm{m}$ toroid plotted versus $d$ is provided in Fig. 15. The calculation uses a finite-element mode solver as there are no analytical expressions available for toroid mode volume. The case of $d=50 \mu \mathrm{m}$ corresponds to a sphere. Overall, the toroidal mode volume can be seen to be lower than the sphere. There are also two distinct regimes of mode volume behavior. In the first, the mode volume reduces very slowly as $d$ is reduced $\left(V \propto d^{1 / 4}\right)$. This regime features a weak lateral confinement very similar in nature to that of the sphere and related to the gentle transverse curvature of the toroidal dielectric boundary. In the second regime, the mode volume reduces very quickly as $d$ is reduced. This regime is characterized by strong, lateral, index confinement resulting from the toroidal boundary being comparable in diameter to the mode field. The inset to this figure provides mode fields for the toroid in these two regimes to further illustrate this idea. It is clear that substantial reductions in threshold are possible using these devices. We are currently characterizing the reductions possible experimentally.

\section{SUMMARY}

We have experimentally and theoretically analyzed Raman oscillation in fiber-taper-coupled microspheres and microtoroids on-a-chip. A theoretical analysis was presented using the coupled mode equations for the pump and Raman WGMs. Using these equations, the threshold condition for stimulated Raman scattering was derived and the relative importance of waveguide coupling strength, mode volume and intrinsic resonator $Q$ were described. These theoretical dependences were verified experimentally. Furthermore the analysis was extended to the case of cascaded Raman oscillation and threshold and efficiency expressions were derived for higher-order Raman fields. This analysis revealed that odd and even order Raman lines exhibit different pump-to-Raman emission characteristics. Even order Stokes fields are found to exhibit a linear increase in generated Raman power as a function of pump power, whereas odd-order Stokes fields exhibit a square root dependence. Analysis showed and experiment confirmed that the threshold for $N$ th-order cascaded Raman oscillation exhibits a cubic dependence on order and that the associated efficiency of the process scales inverse quadratically with order.

Microtoroids, in addition to having significant practical advantages with respect to their chip-based fabrication, have both spectral and power efficiency advantages in comparison to Raman oscillation in microspheres. Their stronger lateral confinement provides two distinct benefits. First, a drastic reduction in the complexity of the mode spectrum enabling single-mode oscillation in the microtoroid based device. Second, a controllable and reduced mode volume so that for comparable $Q$ factors, microtoroid devices should have lower threshold pump powers. We are currently investigating the latter advantage experimentally.

The importance of fiber taper coupling in these measurements cannot be over emphasized. These inherently fiber-compatible waveguides provide exceptional coupling efficiencies to and from the UHQ devices. They are also indispensable in coupling to the microtoroid devices which reside near the surface of a silicon wafer. Using taper coupling, the lowest threshold observed in this study was $62 \mu \mathrm{W}$ of launched power, a value which is nearly three orders of magnitude lower than for free-space illumination of microdroplets.

\section{REFERENCES}

[1] D. W. Vernooy, V. S. Ilchenko, H. Mabuchi, E. W. Streed, and H. J. Kimble, "High-Q measurements of fused-silica microspheres in the near infrared," Opt. Lett., vol. 23, no. 4, pp. 247-249, 1998.

[2] D. W. Vernooy, A. Furusawa, N. P. Georgiades, V. S. Ilchenko, and H. J. Kimble, "Cavity QED with high-Q whispering gallery modes," Phys. Rev. A, Gen. Phys., vol. 57, no. 4, pp. R2293-R2296, 1998.

[3] H. J. Kimble, "Strong interactions of single atoms and photons in cavity QED,” Phys. Scr., vol. T76, pp. 127-137, 1998.

[4] S. Haroche and D. Kleppner, "Cavity quantum electrodynamics," Phys. Today, vol. 42, no. 1, pp. 24-30, 1989. 
[5] V. Sandoghdar, F. Treussart, J. Hare, V. LefevreSeguin, J. M. Raimond, and S. Haroche, "Very low threshold whispering-gallery-mode microsphere laser," Phys. Rev. A, Gen. Phys., vol. 54, no. 3, pp. R1777-R1780, 1996.

[6] M. Cai, O. Painter, K. J. Vahala, and P. C. Sercel, "Fiber-coupled microsphere laser," Opt. Lett., vol. 25, no. 19, pp. 1430-1432, 2000.

[7] Y. Yang, D. K. Armani, and K. J. Vahala, "Fiber-coupled erbium microlasers on a chip," Appl. Phys. Lett., vol. 83, pp. 825-826, 2003.

[8] R. Chang and A. J. Campillo, Optical Processes in Microcavities, Singapore: World Scientific, 1996, vol. 3, Advanced Series in Applied Physics.

[9] S. Uetake, R. S. D. Sihombing, and K. Hakuta, "Stimulated Raman scattering of a high-Q liquid-hydrogen droplet in the ultraviolet region," Opt. Lett., vol. 27, no. 6, pp. 421-423, 2002.

[10] F. Vollmer, D. Braun, A. Libchaber, M. Khoshsima, I. Teraoka, and S. Arnold, "Protein detection by optical shift of a resonant microcavity," Appl. Phys. Lett., vol. 80, no. 21, pp. 4057-4059, 2002.

[11] S. X. Qian, J. B. Snow, H. M. Tzeng, and R. K. Chang, "Lasing droplets-Highlighting the liquid-air interface by laser-emission," Science, vol. 231, no. 4737, pp. 486-488, 1986.

[12] J. Z. Zhang and R. K. Chang, "Generation and suppression of stimulated Brillouin-scattering in single liquid droplets," J. Opt. Soc. Amer.B-Opt. Phys., vol. 6, no. 2, pp. 151-153, 1989.

[13] H. B. Lin and A. J. Campillo, "CW nonlinear optics in droplet microcavities displaying enhanced gain," Phys. Rev. Lett., vol. 73, no. 18, pp. 2440-2443, 1994.

[14] A. J. Campillo, J. D. Eversole, and H. B. Lin, "Cavity quantum electrodynamic enhancement of stimulated- emission in microdroplets," Phys. Rev. Lett., vol. 67, no. 4, pp. 437-440, 1991.

[15] H. B. Lin and A. J. Campillo, "Microcavity enhanced Raman gain," Opt. Commun., vol. 133, no. 1-6, pp. 287-292, 1997.

[16] J. Z. Zhang and R. K. Chang, "Generation and suppression of stimulated Brillouin-scattering in single liquid droplets," J. Opt. Soc. Amer. B-Opt. Phys., vol. 6, no. 2, pp. 151-153, 1989.

[17] V. B. Braginsky, M. L. Gorodetsky, and V. S. Ilchenko, "Quality-factor and nonlinear properties of optical whispering- gallery modes," Phys. Lett. A, vol. 137, no. 7-8, pp. 393-397, 1989.

[18] D. Braunstein, A. M. Khazanov, G. A. Koganov, and R. Shuker, "Lowering of threshold conditions for nonlinear effects in a microsphere," Phys. Rev. A, vol. 53, no. 5, pp. 3565-3572, 1996.

[19] L. Collot, V. Lefevreseguin, M. Brune, J. M. Raimond, and S. Haroche, "Very high-Q whispering-gallery mode resonances observed on fusedsilica microspheres," Europhys. Lett., vol. 23, no. 5, pp. 327-334, 1993.

[20] F. Treussart, V. S. Ilchenko, J. F. Roch, J. Hare, V. Lefevre-Seguin, J. M. Raimond, and S. Haroche, "Evidence for intrinsic kerr bistability of high-Q microsphere resonators in superfluid helium," Eur. Phys. J. D, vol. 1 , no. 3, pp. 235-238, 1998 .

[21] M. L. Gorodetsky and V. S. Ilchenko, "High-Q optical whispering-gallery microresonators-Precession approach for spherical mode analysis and emission patterns with prism couplers," Opt. Commun., vol. 113, no. 1-3, pp. 133-143, 1994.

[22] M. L. Gorodetsky, A. A. Savchenkov, and V. S. Ilchenko, "Ultimate Q of optical microsphere resonators," Opt. Lett., vol. 21, no. 7, pp. 453-455, 1996.

[23] M. L. Gorodetsky and V. S. Ilchenko, "Optical microsphere resonators: Optimal coupling to high-Q whispering-gallery modes," J. Opt. Soc. Amer. B-Opt. Phys., vol. 16, no. 1, pp. 147-154, 1999.

[24] M. L. Gorodetsky, A. D. Pryamikov, and V. S. Ilchenko, "Rayleigh scattering in high-Q microspheres," J. Opt. Soc. Amer. B-Opt. Phys., vol. 17 , no. 6, pp. 1051-1057, 2000.

[25] V. S. Ilchenko, M. L. Gorodetsky, and S. P. Vyatchanin, "Coupling and tunability of optical whispering-gallery modes-A basis for coordinate meter," Opt. Commun., vol. 107, no. 1-2, pp. 41-48, 1994.

[26] V. S. Ilchenko, M. L. Gorodetsky, X. S. Yao, and L. Maleki, "Microtorus: A high-finesse microcavity with whispering-gallery modes," Opt. Lett., vol. 26, no. 5, pp. 256-258, 2001.

[27] J. C. Knight, G. Cheung, F. Jacques, and T. A. Birks, "Phase-matched excitation of whispering-gallery-mode resonances by a fiber taper," Opt. Lett., vol. 22, no. 15, pp. 1129-1131, 1997.

[28] S. M. Spillane, T. J. Kippenberg, O. J. Painter, and K. J. Vahala, "Ideality in a fiber-taper-coupled microresonator system for application to cavity quantum electrodynamics," Phys. Rev. Lett., vol. 91, no. 4, p. 043902 , 2003.

[29] M. Cai, P. O. Hedekvist, A. Bhardwaj, and K. Vahala, "5-Gb/s ber performance on an all fiber-optic add/drop device based on a taper-resonatortaper structure," IEEE Photon. Technol. Lett., vol. 12, pp. 1177-1179, Sept. 2000.
[30] M. Cai, O. Painter, and K. J. Vahala, "Observation of critical coupling in a fiber taper to a silica- microsphere whispering-gallery mode system," Phys. Rev. Lett., vol. 85, no. 1, pp. 74-77, 2000.

[31] S. M. Spillane, T. J. Kippenberg, and K. J. Vahala, "Ultralow-threshold Raman laser using a spherical dielectric microcavity," Nature, vol. 415, no. 6872 , pp. $621-623,2002$.

[32] B. Min, T. Kippenberg, and K. Vahala, "A compact, fiber-compatible cascaded Raman laser,” Opt. Lett., vol. 28, pp. 17-17, 2003.

[33] D. K. Armani, T. J. Kippenberg, S. M. Spillane, and K. J. Vahala, "Ultrahigh-Q toroid microcavity on a chip," Nature, vol. 421, no. 6926, pp. 925-928, 2003.

[34] T. J. Kippenberg, S. M. Spillane, and K. J. Vahala, "Ultralow-threshold microcavity Raman laser on a microelectronic chip," Opt. Lett., vol. 29, no. 11, pp. 1224-1226, 2004

[35] D. S. Weiss, V. Sandoghdar, J. Hare, V. Lefevreseguin, J. M. Raimond, and S. Haroche, "Splitting of high-Q mie modes induced by light backscattering in silica microspheres," Opt. Lett., vol. 20, no. 18, pp. $1835-1837,1995$.

[36] H. A. Haus, Electromagnetic Fields and Energy. Englewood Cliffs, NJ: Prentice-Hall, 1989

[37] T. J. Kippenberg, S. M. Spillane, and K. J. Vahala, "Kerr-nonlinearity optical parametric oscillation in an ultra-high-Q toroid microcavity," Phys. Rev. Lett., vol. 93, no. 8, p. 083 904, 2004.

[38] R. Boyd, Nonlinear Optics. Boston, MA: Academic, 2003

[39] T. J. Kippenberg, S. M. Spillane, and K. J. Vahala, "Modal coupling in traveling-wave resonators," Opt. Lett., vol. 27, no. 19, pp. 1669-1671, 2002.

[40] J. R. Buck and H. J. Kimble, "Optimal sizes of dielectric microspheres for cavity QED with strong coupling," Phys. Rev. A, vol. 67, no. 3, p. $033806,2003$.

[41] B. E. Little, J. P. Laine, and H. A. Haus, "Analytic theory of coupling from tapered fibers and half-blocks into microsphere resonators," $J$. Lightwave Technol., vol. 17, pp. 704-715, Apr. 1999.

[42] A. B. Matsko, A. A. Savchenkov, R. J. Letargat, V. S. Ilchenko, and L. Maleki, "On cavity modification of stimulated Raman scattering," $J$. Opt. B-Quantum Semiclassical Opt., vol. 5, no. 3, pp. 272-278, 2003.

Tobias J. Kippenberg was born in Germany in 1976. He received the undergraduate (Vordiplom) degree in physics and electrical engineering from the Rheinisch Westfaelische Technische Hochschule-Aachen, Aachen, Germany, in 1998, and the M.S. and Ph.D. degrees from the California Institute of Technology (Caltech), Pasadena, in 1999 and 2004, respectively.

He is currently a Postdoctoral Scholar with the Center for the Physics of Information, Caltech.

Dr. Kippenberg is a member of the Studienstiftung des Deutschen Volkes. He was a recipient of a First Prize at the 8th European Union Contest for Young Scientists in Helsinki, Finland, in 1996.

Sean M. Spillane received the degree in engineering physics from Cornell University, Ithaca, NY, in 1998 and the Ph.D. degree from the Department of Applied Physics, California Institute of Technology, Pasadena, in 2004.

$\mathrm{He}$ is currently a Postdoctoral Research Associate with the Quantum Science Research Group, Hewlett-Packard Laboratories, Palo Alto, CA.

Bumki Min, photograph and biography not available at the time of publication.

Kerry J. Vahala (S'82-M'84) received the Ph.D. degree in applied physics from the California Institute of Technology (Caltech), Pasadena, CA, in 1985.

$\mathrm{He}$ is currently a Professor of applied physics at Caltech. His research interests include semiconductor laser and fiber laser physics, high-speed dynamics in semiconductors, and semiconductor nanostructure physics and fabrication.

Dr. Vahala has served as an Associate Editor for IEEE PHOTONICS TECHNOLOGY LETTERS and the Journal of Semiconductor Science and Technology. He has also served as Topical Editor for the Journal of the Optical Society of America. He is the first recipient of the Richard P. Feynman Hughes Fellowship and has also received both the Presidential Young Investigator and Office of Naval Research Young Investigator Awards. In addition, he has been recognized twice by the Student Association at Caltech for excellence in teaching. He is a Fellow of the Optical Society of America. 